\title{
SOME PROPERTIES OF SPECIAL FUNCTIONS DERIVED FROM THE THEORY OF CONTINUOUS TRANSFORMATION GROUPS
}

\author{
MRINAL KANTI DAS
}

\begin{abstract}
The theory of continuous transformation groups is utiltzed in the study of some properties of special functions. On constructing the continuous transformation groups corresponding to a suitably defined infinitesimal transformation, a bilateral generating relation involving Laguerre polynomials $\left\{L_{n}^{(\alpha)}(x)\right\}$ is obtained in $\S 2$. It is shown to be a generalisation of Brafman's result. In the last section raising and lowering operators for $\left\{P_{n}^{(\alpha, \beta-n)}(x)\right\}$ and their commutator are introduced and on showing that they generate a 3-dimensional Lie algebra, the idea of c.t. groups is employed to establish a generating relation involving $\left\{P_{n}^{(\alpha, \beta-n)}(x)\right\}$ which is seen to yield a number of known results. Moreover, a bilateral generating relation involving $\left\{P_{n}^{(\alpha, \beta-n)}(x)\right\}$ is obtained; this is seen to be a generalisation of a well-known relation due to Weisner.
\end{abstract}

1. The purpose of this note is to prove some relations involving the Laguerre polynomials $\left\{L_{n}^{(\alpha)}(x)\right\}$ and the Jacobi polynomials $\left\{P_{n}^{\alpha, \beta-n}(x)\right\}$ by the methods from the theory of continuous transformation groups. (For previous works using these methods see [1], [2], [3], [4], [5].) Our principal results are contained in (2.6), (2.8), (3.7), (3.8) and (3.12).

2. The Laguerre polynomial $L_{n}^{(\alpha)}(x)$ is defined by

$$
L_{n}^{(\alpha)}(x)=\left((1+\alpha)_{n} / n !\right)_{1} F_{1}(-n ; 1+\alpha ; x) .
$$

Let us consider the function

$$
F_{n}(x, t, z)=\exp \left(n+\frac{1}{2}(\alpha+1) t+z-\frac{1}{2} x\right) \cdot x^{(\alpha+1) / 2} L_{n}^{(\alpha)}(x) .
$$

By the help of the well-known recursion relations for $\left\{L_{n}^{(x)}(x)\right\}$, it can be easily checked that the operator $\mathscr{R}=e^{t}\left(\partial / \partial t-\frac{1}{2} x \partial / \partial z-x(\partial / \partial x)\right)$ satisfies

$$
\mathscr{R} F_{n}(x, t, z)=(n+1) F_{n+1}(x, t, z) .
$$

Received by the editors July 6, 1971 .

AMS 1970 subject classifications. Primary 22E99. 
The effects of $(\exp w \mathscr{R})$ upon $x, t, z$ are seen to be [3]

$$
(\exp w \mathscr{R})\left\{\begin{array}{l}
x \\
t \\
z
\end{array}\right\}=\left\{\begin{array}{l}
x^{\prime} \\
t^{\prime} \\
z^{\prime}
\end{array}\right\}=\left\{\begin{array}{c}
x /\left(1-w e^{t}\right) \\
t-\log \left(1-w e^{t}\right) \\
z-x w e^{t} / 2\left(1-w e^{t}\right)
\end{array}\right\} .
$$

Varma recently proved that [6]

$$
\begin{aligned}
(1-w)^{-a} \phi_{1}\left(a ; b, b+\alpha+1 ; \frac{w}{w-1}\right. & \left., \frac{w x}{w-1}\right) \\
= & \sum_{n=0}^{\infty} \frac{(a)_{n}}{(b+\alpha+1)_{n}} w^{n} L_{n}^{(\alpha)}(x),
\end{aligned}
$$

where $|w /(w-1)|<1, \alpha>-1$, and $\phi_{1}(\alpha ; \beta, \gamma ; x, y)$ is the confluent hypergeometric series

$$
\phi_{1}(\alpha ; \beta, \gamma ; x, y)=\sum_{m, n=0}^{\infty} \frac{(\alpha)_{m+n}(\beta)_{n}}{(\gamma)_{m+n} m ! n !} x^{n} y^{m} .
$$

In (2.4), we replace $w$ by wye $e^{t}$ and multiply both sides by

$$
\exp \left\{\frac{1}{2}(\alpha+1) t+z-\frac{1}{2} x\right\} \cdot x^{(\alpha+1) / 2},
$$

so that by $(2.1)$, we have

$$
\begin{aligned}
\left(1-w y e^{t}\right)^{-a} \exp \left\{\frac{1}{2}(\alpha+1) t+z-\frac{1}{2} x\right\} \cdot x^{(\alpha+1) / 2} \\
\\
\times \phi_{1}\left(a ; b, b+\alpha+1 ; w y e^{t} /\left(w y e^{t}-1\right), x w y e^{t} /\left(w y e^{t}-1\right)\right) \\
=\sum_{n=0}^{\infty} \frac{(a)_{n}}{(b+\alpha+1)_{n}} w^{n} y^{n} F_{n}(x, t, z) .
\end{aligned}
$$

On operating both sides of the last equation by $\exp w \mathscr{R}$ and recalling (2.2) and (2.3), we have

$$
\begin{aligned}
\left(1-w y e^{t^{\prime}}\right)^{-a} & \exp \left\{\frac{1}{2}(\alpha+1) t^{\prime}+z^{\prime}-\frac{1}{2} x^{\prime}\right\} \cdot x^{\prime(\alpha+1) / 2} \\
& \times \phi_{1}\left(a ; b, b+\alpha+1 ; w y e^{t^{\prime}} /\left(w y e^{t^{\prime}}-1\right), x^{\prime} w y e^{t^{\prime}} /\left(w y e^{t^{\prime}}-1\right)\right) \\
= & (\exp w \mathscr{R}) \sum_{n=0}^{\infty} \frac{(a)_{n}}{(b+\alpha+1)_{n}} w^{n} y^{n} F_{n}(x, t, z) \\
= & \sum_{m=0}^{\infty} \sum_{n=0}^{\infty} \frac{(a)_{n}}{(b+\alpha+1)_{n}} y^{n} \frac{w^{m+n}}{m !} \mathscr{R}^{m} F_{n}(x, t, z) \\
= & \sum_{m=0}^{\infty} \sum_{n=0}^{\infty} \frac{(m+n) !}{n ! m !} \cdot \frac{(a)_{n}}{(b+\alpha+1)_{n}} y^{n} w^{m+n} F_{m+n}(x, t, z) \\
= & \sum_{n=0}^{\infty} w^{n} F_{n}(x, t, z) \sum_{m=0}^{n}\left(\begin{array}{l}
n \\
m
\end{array}\right) \frac{(a)_{m}}{(b+\alpha+1)_{m}} y^{m} \\
= & \sum_{n=0}^{\infty} w^{n} f_{n}(y) F_{n}(x, t, z)
\end{aligned}
$$


where

$$
f_{n}(y)=\sum_{m=0}^{n}\left(\begin{array}{l}
n \\
m
\end{array}\right) \frac{(a)_{m}}{(b+\alpha+1)_{m}} y^{m}={ }_{2} F_{1}\left[\begin{array}{c}
-n, a ; \\
b+\alpha+1 ;
\end{array}-y\right] .
$$

On substituting the values of $x^{\prime}, t^{\prime}, z^{\prime}$ in (2.5) and on putting $t=z=0$, the relation (2.5) becomes

$$
\begin{aligned}
& \sum_{n=0}^{\infty} w_{2}^{n} F_{1}\left[\begin{array}{r}
-n, a ; y] L_{n}^{(\alpha)}(x) \\
b+\alpha+1 ;
\end{array}\right] \\
& =\exp \left(\frac{-x w}{1-w}\right) \cdot(1-w)^{a-\alpha-1}(1-w+y w)^{-a} \\
& \quad \times \phi_{1}(a ; b, b+\alpha+1 ; \\
& \quad y w /(1-w+y w), x y w /(1-w+w y)(1-w)),
\end{aligned}
$$

which is noteworthy.

This result may be considered as a generalisation of the following relation of Brafman [9]:

$$
\begin{aligned}
\sum_{0}^{\infty}{ }_{2} F_{1}\left[\begin{array}{l}
-n, c ; y \\
1+\alpha ;
\end{array}\right] L_{n}^{(\alpha)}(x) w^{n} & \\
= & \exp (-x w /(1-w)) \cdot(1-w)^{c-\alpha-1} \cdot(1-w+w y)^{-c} \\
& \times{ }_{1} F_{1}\left[\begin{array}{c}
c ; \frac{x y w}{1+\alpha ;(1-w+w y)(1-w)}
\end{array}\right] .
\end{aligned}
$$

We can easily obtain this from (2.6) by the substitution $b=0$.

Now we consider some more particular cases of (2.6). It is known that Jacobi polynomial $P_{n}^{(\mu, \nu)}(z)$ admits of the following explicit evaluation [7]

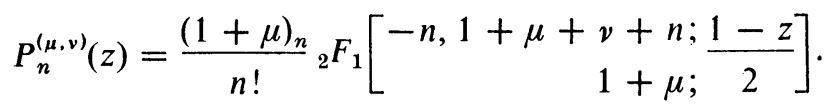

Let us put in (2.6) $b=\mu-\alpha, a=1+\mu+\nu, y=(1-z) / 2$; then from (2.6) follows the bilateral generating relation stated below:

$$
\begin{aligned}
& \begin{aligned}
\sum_{n=0}^{\infty} \frac{n ! w^{n}}{(1+\mu)_{n}} & P_{n}^{(\mu, v-n)}(z) L_{n}^{(\alpha)}(x) \\
= & \exp (-x w /(1-w))(1-w)^{\mu+v-\alpha}\left(1-\frac{1}{2}(1+z) w\right)^{-1-\mu-v} \\
& \times \phi_{1}(1+\mu+v ; \mu-\alpha, \mu+1 ;
\end{aligned} \\
& \quad \frac{\left.\frac{w(1-z)}{2-w)}, \frac{(1-z) x w}{(2-w-z w)(1-w)}\right) .}{2-w w}
\end{aligned}
$$

This seems to be new. 
We note that $\phi_{1}(\alpha ; \beta, \gamma ; x, y)={ }_{2} F_{1}\left[{ }^{\alpha,}{ }_{\gamma} \beta ; y\right]$ when $x=0$, and that $L_{n}^{(\alpha)}(0)=(1+\alpha)_{n} / n !$, and so the relation $(2.8)$ becomes

$$
\begin{aligned}
\sum_{n=0}^{\infty} \frac{(1+\alpha)_{n}}{(1+\mu)_{n}} w^{n} P_{n}^{(\mu, v-n)}(z)= & (1-w)^{\mu+v-\alpha}\left(1-\frac{1}{2}(1+z) w\right)^{-1-\mu-v} \\
& \times{ }_{2} F_{1}\left[\begin{array}{r}
1+\mu+\nu, \mu-\alpha ; \frac{w(1-z)}{1+\mu ;} \\
1+w-z w
\end{array}\right]
\end{aligned}
$$

which may be compared with the result due to Varma [6] stated in $\S 3$.

3. The Jacobi polynomial $P_{n}^{(\alpha, \beta)}(x)$ of degree $n$ is defined in (2.7). In this section we shall find some generating relations for $P_{n}^{(\alpha, \beta-n)}(x)$.

For our convenience, we consider the sequence of polynomials $\left\{\phi_{n}(z)\right\}$ defined by

$$
\phi_{n}(z)=\frac{(1+b)_{n}}{n !}{ }_{2} F_{1}\left[\begin{array}{r}
-n, 1+a ; \\
1+b ;
\end{array}-z\right] .
$$

This sequence obeys the relations

$$
\begin{aligned}
{[(a+1) z+(n+b+1)+z(z+1)(d / d z)] \phi_{n}(z) } & =(n+1) \phi_{n+1}(z), \\
{[-n+z(d / d z)] \phi_{n}(z) } & =-(b+n) \phi_{n-1}(z) .
\end{aligned}
$$

The last two relations show that if we introduce the function $F_{n}(x, t, z)$ : $F_{n} \equiv F_{n}(x, t, z)=\exp (y+n t) \phi_{n}(z)$ and the operators $\mathscr{R}, \mathscr{L}$ :

(3.2a) $\mathscr{R} \equiv e^{t}[\{(a+1) z+(b+1)\} \partial / \partial y+\partial / \partial t+z(z+1) \partial / \partial z]$,

(3.2b) $\mathscr{L} \equiv e^{-t}[-\partial / \partial t+z \partial / \partial z]$,

then we obtain the following relations:

$$
\mathscr{R} F_{n}=(n+1) F_{n+1}, \quad \mathscr{L} F_{n}=-(b+n) F_{n-1}, \quad \mathscr{L} F_{0}=0 ;
$$

thus $\mathscr{R}, \mathscr{L}$ behaving as the raising and lowering operators for the system $\left\{F_{n}(x, t, z)\right\}$ respectively. We see that

$$
\begin{aligned}
& {[\mathscr{R} \mathscr{L}]=\mathscr{R} \mathscr{L}-\mathscr{L} \mathscr{R}=2 \partial / \partial t+(b+1) \partial / \partial y \equiv X,} \\
& {[X \mathscr{R}]=-2 \mathscr{R}, \quad[X \mathscr{L}]=2 \mathscr{L} .}
\end{aligned}
$$

Hence $\mathscr{R}, \mathscr{L}, X$ are the basis of a 3-dimensional Lie algebra which is isomorphic to the Lie algebra of the 3-dimensional rotation group. Consequently, if $Y=p \mathscr{L}+q \mathscr{R}+r X$, then

$$
\begin{aligned}
\exp Y & =\exp (p \mathscr{L}+q \mathscr{R}+r X) \\
& =(\exp u \mathscr{L})(\exp v \mathscr{R})(\exp \tau X)
\end{aligned}
$$


where $u, v, \tau$ are expressible as functions of $p, q, r$ as follows [3]:

$$
\begin{aligned}
e^{-\tau} & =\cosh s-(r / s) \sinh s \\
u & =(p \tanh s) /(s-r \tanh s), \\
w & =(q / s) \cosh s \sinh s-\left(q r / s^{2}\right) \sinh ^{2} s, \quad s=\left(r^{2}-p q\right)^{1 / 2} .
\end{aligned}
$$

Now the continuous transformation groups corresponding to the infinitesimal transformations $\mathscr{R}$ and $Y$ are

$(\exp w \mathscr{R})\left\{\begin{array}{l}z \\ t \\ y\end{array}\right\}=\left\{\begin{array}{l}z^{\prime} \\ t^{\prime} \\ y^{\prime}\end{array}\right\}$

$$
=\left\{\begin{array}{c}
z /\left\{1-(z+1) w e^{t}\right\} \\
t-\log \left(1-w e^{t}\right) \\
y-(b-a) \log \left(1-w e^{t}\right)-(a+1) \log \left(1-w e^{t}(1+z)\right)
\end{array}\right\},
$$

$$
(\exp Y)\left\{\begin{array}{l}
z \\
t \\
y
\end{array}\right\}=\left\{\begin{array}{l}
z^{\prime \prime} \\
t^{\prime \prime} \\
y^{\prime \prime}
\end{array}\right\},
$$

where

$$
\begin{aligned}
z^{\prime \prime}= & z /\left(1-u e^{-t}\right)\left[1-\left(z+1-u e^{-t}\right) v e^{t}\right] \\
t^{\prime \prime}= & t+\log \left(1-u e^{-t}\right)-\log \left(1+u v-v e^{t}\right)+2 \tau, \\
y^{\prime \prime}= & y-(b-a) \log \left(1+u v-v e^{t}\right) \\
& -(a+1) \log \left[1-v e^{t}\left(1-u e^{-t}+z\right)\right]+(b+1) \tau,
\end{aligned}
$$

$u, v, \tau$ being the same as in (3.5).

Remembering the relation (3.4) and the fact that $Y$ is an infinitesimal transformation, we apply the operator $\exp Y$ to the function $F_{m}(z, y, t)$ :

$$
\begin{aligned}
F_{m}\left(z^{\prime \prime}, y^{\prime \prime}, t^{\prime \prime}\right) & =(\exp Y) F_{m}(z, y, t) \\
= & (\exp u \mathscr{L})(\exp v \mathscr{R})(\exp \tau X) F_{m}(z, y, t) \\
= & \exp \tau(2 m+b+1) \cdot(\exp u \mathscr{L})(\exp v \mathscr{R}) \cdot F_{m}(z, y, t) \\
= & \exp \tau(2 m+b+1) \sum_{n=0}^{\infty}\left(\begin{array}{c}
m+n \\
n
\end{array}\right) v^{n} \sum_{k=0}^{m+n} \frac{u^{k} \mathscr{L}^{k}}{k !} F_{m+n}(z, y, t) \\
= & \exp \tau(2 m+b+1) \sum_{n=0}^{\infty}\left(\begin{array}{c}
m+n \\
n
\end{array}\right) v^{n} \\
& \times \sum_{k=0}^{m+n}\left(\begin{array}{c}
b+m+n \\
k
\end{array}\right)(-u)^{k} \phi_{m+n-k}(z) \exp \{y+(m+n-k) t\}
\end{aligned}
$$

where $\left(\begin{array}{l}p \\ k\end{array}\right)$ stands for $p(p-1) \cdots(p-k+1) / k$ !. 
Putting $y=t=0$ and using (3.6b) we get

$$
\begin{aligned}
(1-u)^{m}(1+u v-v)^{-m-b+a}\{1-v(1-u+z)\}^{-a-1} \\
\times \phi_{m}(z /(1-u)(1-(1+z+u) v)) \\
=\sum_{n=0}^{\infty}\left(\begin{array}{c}
m+n \\
n
\end{array}\right) v^{n} \sum_{k=0}^{m+n}(-u)^{k}\left(\begin{array}{c}
b+m+n \\
k
\end{array}\right) \phi_{m+n-k}(z) .
\end{aligned}
$$

We recall the definition of $\phi_{n}(z)$ in (3.1) and replace $u$ by $-u$, so that the above relation gives

$$
(1+u)^{m}(1-u v-v)^{-m-b+a}\{1-v(1+u-z)\}^{-a-1}
$$

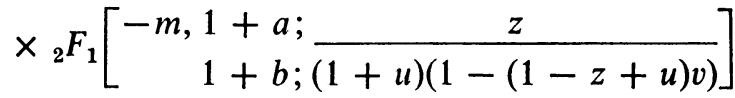

$$
\begin{aligned}
& =\sum_{n=0}^{\infty} \frac{(1+b)_{m+n}}{(1+b)_{m}} \cdot \frac{v^{n}}{n !} \sum_{k=0}^{m+n} u^{k}\left(\begin{array}{c}
m+n \\
k
\end{array}\right){ }_{2} F_{1}\left[\begin{array}{r}
-m-n+k, 1+a ; z \\
1+b ;
\end{array}\right] \text {. }
\end{aligned}
$$

This is noteworthy.

Let us put $a=\alpha+\beta, b=\alpha, z=(1-x) / 2$ in (3.7) and remember (3.1); then we have

$$
\begin{aligned}
(1+u)^{m} & (1-u v-v)^{-m+\beta}\{1-v(u+(1+x) / 2)\}^{-\alpha-\beta-1} \\
& \times P_{m}^{(\alpha, \beta-m)}(1-(1-x) /(1+u)(1-(u+(1+x) / 2) v)) \\
= & \sum_{n=0}^{\infty}\left(\begin{array}{c}
m+n \\
n
\end{array}\right) v^{n} \sum_{k=0}^{m+n}\left(\begin{array}{c}
\alpha+m+n \\
k
\end{array}\right) u^{k} P_{m+n-k}^{(\alpha, \beta-m-n+k)}(x) .
\end{aligned}
$$

The formulae (3.7) and (3.8) are new. They contain previously derived results as special cases: the cases $u=0$ of (3.7), (3.8) are known, so are the cases $v=0$.

Next we note Varma's result [6]:

$$
\begin{aligned}
& \sum_{n=0}^{\infty} \frac{(a)_{n}}{(b+\alpha+1)_{n}} w^{n} P_{n}^{(\alpha, \beta-n)}(x) \\
& \quad=(1-w)^{-a} F_{1}\left(a, b, 1+\alpha+\beta, 1+\alpha+b ; \frac{-w}{1-w}, \frac{-w(1-x)}{2(1-w)}\right),
\end{aligned}
$$

which we can write as follows:

$$
\begin{aligned}
\sum_{n=0}^{\infty} \frac{(\alpha)_{n}}{(b+\beta+1)_{n}} & w^{n} \phi_{n}(z) \\
& =(1-w)^{-\alpha} F_{1}\left(\alpha, \beta, 1+a, 1+b+\beta ; \frac{-w}{1-w}, \frac{w z}{1-w}\right) .
\end{aligned}
$$


Replacing $w$ by $x w e^{t}$ and multiplying both sides by $e^{y}$, we have

$$
\begin{aligned}
& \sum_{n=0}^{\infty} \frac{(\alpha)_{n}}{(b+\beta+1)_{n}} w^{n} x^{n} F_{n}(z, y, t) \\
& \quad=e^{y}\left(1-x w e^{t}\right)^{-\alpha} F_{1}\left(\alpha, \beta, 1+a, 1+b+\beta ; \frac{-x w e^{t}}{1-x w e^{t}}, \frac{x w z e^{t}}{1-x w e^{t}}\right)
\end{aligned}
$$

Operating both sides of (3.9) by $(\exp w \mathscr{R})$, we get, by virtue of (3.3), $(\exp w \mathscr{R}) \cdot e^{y}\left(1-x w e^{t}\right)^{-\alpha}$

$$
\begin{aligned}
& \times F_{1}\left(\alpha, \beta, 1+a, 1+b+\beta ; \frac{-x w e^{t}}{1-x w e^{t}}, \frac{x w e^{t} z}{1-x w e^{t}}\right) \\
= & (\exp w \mathscr{R}) \cdot \sum_{n=0}^{\infty} \frac{(\alpha)_{n}}{(b+\beta+1)_{n}} w^{n} x^{n} F_{n}(z, y, t) \\
= & \sum_{m=0}^{\infty} \sum_{n=0}^{\infty} \frac{(\alpha)_{n} w^{n+m} x^{n}}{(b+\beta+1)_{n}} \cdot \frac{\mathscr{R}^{m}}{m !} F_{n}(z, y, t) \\
= & \sum_{m=0}^{\infty} \sum_{n=0}^{m} \frac{w^{m} x^{n}(\alpha)_{n}}{(b+\beta+1)_{n}} \cdot \frac{m !}{n !(n-m) !} F_{m}(z, y, t) \\
= & \sum_{m=0}^{\infty} w^{m} F_{m}(z, y, t) f_{m}(x)
\end{aligned}
$$

where

$$
\begin{aligned}
f_{m}(x) & =\sum_{n=0}^{\infty}\left(\begin{array}{c}
m \\
n
\end{array}\right) \frac{(\alpha)_{n}}{(b+\beta+1)_{n}} x^{n} \\
& =\sum_{n=0}^{\infty} \frac{(-m)_{n}(\alpha)_{n}}{(b+\beta+1)_{n}} \cdot \frac{(-x)^{n}}{n !} \\
& ={ }_{2} F_{1}\left[\begin{array}{c}
-m, \alpha ; \\
b+\beta+1 ;
\end{array}\right] .
\end{aligned}
$$

Thus from (3.10), (3.11), (3.2) and (3.6a), we have

$$
\begin{aligned}
& \sum_{m=0}^{\infty} \frac{(1+b)_{m}}{m !} \exp (y+m t) w^{m}{ }_{2} F_{1}\left[\begin{array}{r}
-m, 1+a ; \\
1+b ;
\end{array}-z{ }_{2} F_{1}\left[\begin{array}{c}
-m, \alpha ; \\
b+\beta+1 ;
\end{array}\right]\right. \\
& =e^{y^{\prime}}\left(1-x w e^{t^{\prime}}\right)^{-\alpha} F_{1}\left(\alpha, \beta, 1+a, 1+b+\beta ; \frac{-x w e^{t^{\prime}}}{1-x w e^{t^{\prime}}}, \frac{x w z^{\prime} e^{t^{\prime}}}{1-x w e^{t^{\prime}}}\right)
\end{aligned}
$$

which becomes, on substituting the values of $z^{\prime}, y^{\prime}, t^{\prime}$ from (3.6a) and 
thereafter putting $y=t=0$ and replacing $x, z$ by $-x,-z$ respectively

$$
\begin{gathered}
\sum_{m=0}^{\infty} \frac{(1+b)_{m}}{m !} w^{m}{ }_{2} F_{1}\left[\begin{array}{r}
-m, 1+a ; z \\
1+b ;
\end{array}\right]{ }_{2} F_{1}\left[\begin{array}{r}
-m, \alpha ; x \\
b+\beta+1 ;
\end{array}\right] \\
=(1-w)^{a-b+\alpha}(1-w+w z)^{-a-1}(1-w+w x)^{-\alpha} \\
\quad \times F_{1}(\alpha, \beta, 1+a, 1+b+\beta
\end{gathered}
$$

$$
\left.\frac{x w}{1-w+w x}, \frac{x w z}{(1-w+w z)(1-w+w x)}\right) \text {. }
$$

A particular case of the relation (3.12) is noteworthy: putting $\beta=0$ and changing $a, b$ to $a-1, b-1$ respectively, we get the following result proved by Weisner [8]:

$$
\begin{aligned}
& \sum_{m=0}^{\infty} \frac{(b)_{m}}{m !} w_{2}^{m} F_{1}\left[\begin{array}{r}
-m, a ; z \\
b ;
\end{array}\right]{ }_{2} F_{1}\left[\begin{array}{r}
-m, \alpha ; x \\
b ;
\end{array}\right] \\
& =(1-w)^{a-b+\alpha}(1-w+w z)^{-a}(1-w+w x)^{-\alpha}
\end{aligned}
$$

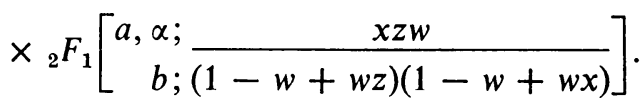

Thus our result (3.12) may be considered to be a generalisation of this result of Weisner.

Making a simple change of parameters and also of the variables $x, z$ we see that the following relation is evident from (3.12):

$$
\begin{aligned}
& \sum_{m=0}^{\infty} \frac{m !}{(1+\beta)_{m}} w^{m} P_{m}^{(b, a-m)}(z) P_{m}^{(\beta, \alpha-m)}(x) \\
& =(1-w)^{a+\alpha+\beta+1}\{1-(1+z) w / 2\}^{-a-b-1}\{1-(1+x) w / 2\}^{-\alpha-\beta-1} \\
& \quad \times F_{1}(1+\alpha+\beta, \beta-b, 1+a+b, 1+\beta ; \\
& \left.\quad \frac{(1-x) w / 2}{1-(1+x) w / 2}, \frac{(1-x)(1-z) w / 4}{(1-(1+x) w / 2)(1-(1+z) w / 2)}\right) .
\end{aligned}
$$

This may be compared with a result due to Srivastava [10].

\section{REFERENCES}

1. B. Kaufman, Special functions of mathematical physics from the viewpoint of Lie algebra, J. Mathematical Phys. 7 (1966), 447-457. MR 33 \#5951.

2. S. K. Chatterjea, Quelques fonctions génératrices des polynômes d'Hermite du point vue l'algèbre de Lie, C. R. Acad. Sci. Paris Sér. A-B 268 (1969), A600-A604. MR 39 \#1079. 
3. M. K. Das, Sur les polynômes de Laguerre, du point de vue de l'algèbre de Lie, C. R. Acad. Sci. Paris Sér. A-B 270 (1970), A380-A383. MR 41 \#2093.

4. - Sur les polynômes d'Hermite, du point de vue de l'algèbre de Lie, C. R. Acad. Sci. Paris Sér. A-B 270 (1970), A452-A455. MR 41 \#3845.

5. —- Sur les polynômes de Bessel, du point de vue de l'algèbre de Lie, C. R. Acad. Sci. Paris Sér. A-B 271 (1970), A361-A364. MR 42 \#3330.

6. V. K. Varma, Double hypergeometric functions as generating functions of the Jacobi and Laguerre polynomials, J. Indian Math. Soc. 32 (1968), 1-5. MR 39 \#3064.

7. E. D. Rainville, Special functions, 3rd ed., Macmillan, New York, 1965.

8. L. Weisner, Group-theoretic origin of certain generating functions, Pacific J. Math. 5 (1955), 1033-1039. MR 19, 264.

9. F. Brafman, Some generating functions for Laguerre and Hermite polynomials, Canad. J. Math. 9 (1957), 180-187. MR 19, 28.

10. H. M. Srivastava, Some bilinear generating functions, Proc. Nat. Acad. Sci. U.S.A. 64 (1969), 462-465. MR 42 \#2052.

Center of Advanced Study in Applied Mathematics, 92 Acharya Prafulla Chandra Rd., Calcutta 9, India 